• 论坛・

\title{
IPBES工作效率和科学职能的有效性分析
}

\author{
戴逢斌，吴杨，潘玉雪，张博雅，田瑜*
}

中国环境科学研究院, 北京 100012

\begin{abstract}
摘要: IPBES第一轮工作方案受到国际社会的广泛关注，奠定了其作为第一个生物多样性领域政府间机制的重要地位。IPBES 自成立以来相继发布了系列评估报告和决策者摘要，引起了国际社会广泛关注，因此，其公平性、公正性、科学性和透明性 始终为各界关注的焦点，直接决定着IPBES评估报告的可信度和未来的发展。为有效提升工作效率，提高其成果的科学性， IPBES通过定期开展内部和外部审查，发现问题，优化机构设置，从而指导未来工作计划。本文以IPBES定期开展的审查以及 现有工作机制为基础，针对IPBES的工作效率和科学职能进行分析，从根本上认识其地位和性质，分析其机构设置的优势和 存在的问题，以及工作职能的发挥潜力，针对存在的问题提出建议。并根据我国情况，提出相关工作建议。总体来讲, IPBES 在完善组织机构和规则程序，推动产生新知识、财务资源有效管理等方面均取得了显著进展，但在透明度、科学与政策衔接、 学科和地域平衡，以及政策支持方面存在一定不足，在发挥成员国积极性和能力方面存在欠缺。为此，建议IPBES未来能够更 进一步发挥其特殊的政府间地位和作用，促进科学职能的发挥，特别是加强科学和政策的互动，推动多利益做关方参与，形 成稳定的财务制度并提升工作机制的透明度。同时，建议加强对IPBES评估报告在国内的解读，加大国内宣传力度，并加强多 学科的专家遴选, 弥补研究领域的国内空缺。
\end{abstract}

关键词: 生物多样性; 生态系统服务; 审查; 职能; IPBES

戴逢斌, 吴杨, 潘玉雪, 张博雅, 田瑜 (2021) IPBES工作效率和科学职能的有效性分析. 生物多样性, 29, 688-692. doi: 10.17520/biods.2020250. Dai FB, Wu Y, Pan YX, Zhang BY, Tian Y (2021) Work efficiency of IPBES and the effectiveness of scientific functions. Biodiversity Science, 29, 688-692. doi: 10.17520 /biods.2020250

\section{Work efficiency of IPBES and the effectiveness of scientific functions}

Fengbin Dai, Yang Wu, Yuxue Pan, Boya Zhang, Yu Tian*

Chinese Research Academy of Environmental Sciences, Beijing 100012

\begin{abstract}
Background: The first work programme of IPBES has received extensive attention from the international community, laying down its important role as the first intergovernmental mechanism in the field of biodiversity. Since its establishment, IPBES has successively released a series of assessment reports and summary for policymakers, which have attracted widespread attention from the international community. Therefore, its fairness, impartiality, scientificity and transparency have always been the focus of attention from all walks of life, and directly determine the credibility and future development of IPBES assessment report. In order to effectively improve the scientificity and efficiency of IPBES deliverables, IPBES conducts internal and external reviews regularly to identify problems, optimize institutional settings, and guide future work plans.

Problems: IPBES has made significant progress in improving its organizational structure, rules and procedures, promoting the generation of new knowledge, and the effective management of financial resources. However, there are certain shortcomings in transparency, science and policy linkage, discipline and geographical balance, and policy support. And there are deficiencies in stimulating the enthusiasm and capacity of member states.

Recommendations: To further exert IPBES's special intergovernmental status and role in the future to promote scientific functions, especially to strengthen the interaction between science and policy, it is needed for IPBES to promote multi-stakeholder participation, to form a stable financial system, and to enhance the transparency of its
\end{abstract}

收稿日期: 2020-06-22; 接受日期: 2020-10-09

基金项目: 生态环境部生物多样性保护专项(22110404001)

* 通讯作者 Author for correspondence. E-mail: tianyu@craes.org.cn 
working mechanisms. As to China, it is suggested to strengthen the domestic interpretation of the IPBES assessment report, increase publicity, and strengthen the selection of multidisciplinary experts to fill up the domestic vacancies in the research field.

Key words: biodiversity; ecosystem services; reviews; functions; IPBES

生物多样性和生态系统服务政府间科学政策 平台(Intergovernmental Science-Policy Platform on Biodiversity and Ecosystem Services, IPBES)是连接 科学界和决策者之间的桥梁(田瑜等, 2015)。IPBES 包含创造新知识、开展评估、政策支持和能力建设 四项基本职能(田瑜等, 2016), 通过其四个相互关联 的职能, 缩小科学界与政治界对生物多样性领域的 认识差距，增强对话，提升全球环境的可持续性 (Duke et al, 2015)。自2012年IPBES成立以来，第一 轮工作方案在增进关于生物多样性和生态系统服 务的知识方面已经取得了显著成效, 相继发布了 “传粉者、传粉和粮食生产” “生物多样性和生态系 统服务情景和模型方法” “土地退化和恢复”评估以 及四大地理区域评估和全球评估报告及其决策者 摘要(潘玉雪等, 2020), 引起了国际社会广泛关注, 对扩大国际影响产生了积极效应。因此, 其公平性、 公正性、科学性和透明性始终为各界关注的焦点, 直接决定着IPBES评估报告的可信度和未来的发 展。为此, IPBES将针对工作和科学职能有效性的审 查作为其工作方案的目标之一(IPBES, 2014a)。

根据IPBES的总体目标和任务, 审查内容主要 包括行政和科学职能两个方面(IPBES, 2014b)。科学 职能的发挥直接影响着评估报告的科学可信度, 而 行政职能的发挥则对评估报告的影响力、可信度有 着更为深远的潜在影响。本文在IPBES第一轮工作 方案中开展的内部和外部审查的基础上, 针对 IPBES的工作效率和科学职能进行分析, 从根本上 认识IPBES的地位和性质, 分析其机构设置的优势 和存在的问题, 以及工作职能的发挥潜力, 进而推 测其未来的发展方向。

\section{1 审查的背景和方法}

IPBES的审查是指对工作方案中行政及科学职 能的分析和评估。2013-2017年, IPBES通过制定一 套审查机制(IPBES, 2014a，2015), 对其正在开展的 第一轮工作方案进行了评估分析。审查内容包括 IPBES四项职能的履行、运作原则的应用、制定交
付品程序的成效、机制安排的成效、工作团队和专 家组的成效、预算和财政规划成效等 6 个方面 (IPBES, 2017a)。审查形式分为内部审查及外部审查 (IPBES, 2015)。2015年和2017年, IPBES两个审查小 组分别向全体会议递交了审查报告(IPBES，2015, 2017a), 对下一个工作计划的补充制定和实施具有 重要的指导意义。

内部审查即自查, 由IPBES主席、1名主席团成 员、多学科专家组的 1 名共同主席和 2 名成员, 以及 执行秘书组成内部审查小组, 通过调查问卷开展工 作(IPBES, 2015)。内部审查小组根据收到的85份不 同身份人员 $(9$ 名主席团成员、 23 名多学科专家组成 员、 21 名国家联络人、17名IPBES专家和17名秘书 处成员，包括技术支持机构成员)填写的问卷进行 自我评估，编制内部审查报告(IPBES, 2017b)。审查 报告在2017年的第六次全体会议上获得通过。

外部审查小组由 10 名独立专家组成, 由竞争性 招标程序遴选出的外部独立审查机构协助开展工 作(IPBES, 2017b)。通过问卷调查、分析发表文章情 况及引用数、在线资源访问情况、关键人物访谈等 方式, 外部审查小组收集了主席团、多学科专家组 成员、秘书处、利益敒关方、国家联络点以及专家 等反馈的信息(IPBES, 2017b), 编写了审查报告, 在 2019年的IPBES第七次全体会议上获得通过(IPBES, 2019b)。

\section{2 审查的内容和结论}

IPBES成立以来, 利用有限的资源制定了必要 的议事规则, 取得了一系列成果, 相继发布了系列 评估报告和决策者摘要, 引起了国际社会的广泛关 注, 成功推动了国际社会对生物多样性和生态系统 服务现状和趋势的了解(潘玉雪等, 2020)。但与此同 时, IPBES仍面临着诸多挑战, 如与政策衔接不够 紧密、财政来源不稳定、四大职能进展不同步等 (IPBES, 2019a)。

\section{1 行政职能的有效性}

在联合国环境规划署的推动下, 经过长期的国 
际谈判和讨论, IPBES于 2012年正式成立(马克平, 2012)。作为一个新的机构, 其行政管理模式借鉴了 IPCC 的方式, 但其行政职能的有效性仍存在以下 不足。

第一，政府间机制的作用正逐步发挥，但法律 地位和远期目标仍待进一步明确。作为一个独立的 政府间机制，其成员为各国政府，同时联合国机 构、联合国环境公约和非政府组织通过建立合作伙 伴关系的形式参与(IPBES, 2019a)。然而, IPBES在 定位和远期目标方面，仍然存在一些问题和挑战: (1) IPBES的法律地位仍需提高, 从而提升IPBES履 行总体任务和影响政策的能力; (2)利益做关方甚至 成员国对IPBES的法律地位存在认识混乱的问题, 仍将其定位成一个联合国机构，而不是一个政府间 平台, 对其促进科学政策互动的政府间作用的认识 含糊不清; (3)对未来愿景和使命没有清晰的勾画, 战略上重视不够。IPBES在成立之初设定的目标为 “通过建立科学与政策之间的联系, 加强生物多样 性保护与可持续利用, 确保长期的人类福祉和可持 续发展” (IPBES, 2014a)。部分成员认为, IPBES应该 有一个可以衡量的战略性目标, 以便有效地指导和 推进各个阶段的工作方案。此外, 性别平衡、地域 平衡及学科(尤其是社会科学)平衡仍需进一步提 升。

第二, 体制机制初步完善, 但尚不成熟。迄今 为止, IPBES建立了全面的运作管理和工作机制, 并制订了一系列的议事规则, 其秘书处及技术支持 机构的工作也受到了各方肯定(IPBES, 2019a)。特别 是IPBES针对各项职能设立了技术支持机构和特别 工作组(IPBES, 2014a), 引导和促进利益做关方参 与, 吸纳土著和地方知识及其他知识体系, 建立审 查工作机制，有效促进了 IPBES的可持续性和长期 影响。但仍然存在一些问题, 如IPBES当前的体制 结构设计过于繁琐, 多学科专家组和主席团的职责 存在重叠, 经常造成重复工作和职责划分不明确的 情况; 秘书处工作负担过重, 其技术支持机构发挥 作用需要一定时间, 而这些工作人员的地位不高, 影响力不大; 尽管IPBES已经认识到利益做关方参 与工作的重要性, 但由于实施存在困难导致其发挥 的作用有限。此外, IPBES对联合国四个组织(联合 国粮农组织、联合国开发计划署、联合国环境规划 署、联合国教科文组织)及若干多边环境协定合作的
潜在价值未能充分发挥，协同合作仍有待加强 (IPBES, 2019a)。

第三，资金管理高效，但缺乏稳定来源。总体 上, IPBES对现有可用资源的管理合理高效。大约一 半资源用于工作方案的执行，且其中大部分资源用 于较为重要的区域和全球评估; 另一半资源用于维 持IPBES的运作和管理。但是，由于IPBES资金机制 是成员国自愿捐款，因此一直以来缺乏稳定可靠的 长期收入来源, 财务容易受各国捐款数额波动的影 响，在可持续筹资方面也难以取得重大进展(IPBES, 2019a)。因此, IPBES不得不对财务资源不定期进行 调整，工作方案的启动也采用了灵活多变的滚动方 式, 这对IPBES的财务管理造成了较大压力。

\section{2 科学职能的有效性}

IPBES成立以来, 在评估知识状况、推动创造 新知识、开展能力建设等方面取得了成功。IPBES 发布的科学评估报告和相关的决策者摘要数量众 多，先后发布了“传粉者、传粉和粮食生产” “生物多 样性和生态系统服务情景和模型” “土地退化和恢 复”“四大地理区域评估”和全球评估报告决策者摘 要等重要成果(潘玉雪等, 2020), 引起了国际社会的 广泛关注。同时, IPBES接纳所有知识来源, 不仅包 括科学研究成果, 还包括土著和地方知识, 并鼓励 利益敒关方参与, 且尝试在其产出中考虑文化差异 (IPBES, 2019a)。IPBES评估报告一定程度上提升了 各种知识持有者和政策制定者的理解和能力, 推动 通过知识与政策的衔接来传递知识。可以说, IPBES 科学职能的发挥相较于行政职能效果更为显著。

然而, IPBES在科学职能的发挥上仍存在一些 问题: (1)为确保公平性, 实现联合国五个区域的地 域平衡、发达国家和发展中国家的平衡、性别平衡 等, 在某种程度上导致评估团队在学科和知识水平 方面的参差不齐(IPBES，2019a); (2)虽然鼓励土著 和地方知识纳入IPBES, 但由于缺少实践经验, 还 未形成完善的工作机制，一定程度上阻碍了土著和 地方知识持有者参与工作 (IPBES, 2019a); (3)在邀 请生物多样性领域外专家参与评估方面也存在困 难, 由于专家笁选机制不透明, 专家资质、要求及 篮选过程不公开, 导致在部分学科出现空白, 政府 的参与力度有限, 这也对IPBES实现其既定目标造 成了负面影响; (4)在与IPBES知识和数据管理有关 的职能方面，由于收集和使用基础信息和数据的标 
准和导则始终没有出台, 导致评估过程中的信息和 文献没有得到有效收集和存储, 为后续专家和政府 评审工作带来了不便, 极大影响了科学可信度。

\section{3 政策影响力}

IPBES的第一轮工作方案虽然得到了成员国的 普遍肯定, 同时也得到了学术界、环境和生物多样 性保护有关的国际机构和非政府组织的支持, 但由 于IPBES早期开展评估时很大程度上是作为一个科 学组织来进行, 侧重于建立其科学和技术信誉, 没 有充分驾驭科学与政策之间的衔接, 因此, 政策支 持职能实施得不理想, 评估报告决策者摘要未对决 策者产生预期的影响(IPBES, 2019a)。此外, 受语 言、文化、传播时间、理解能力及其他信息源等的 影响, 这也在一定程度上限制了各国在政策层面对 其评估结果的理解和应用。

\section{IPBES未来的机遇和挑战}

2019年, 达沃斯经济论坛在审议年度《全球风 险报告》时, 突出强调了环境恶化对商业和人类社 会的风险，报告指出了气候变化、生物多样性丧失 和生态系统崩溃等重要环境问题, 特别是它们之间 的关联和反馈，引起国际社会政商界领导人的重视， 迫切需要清楚了解生物多样性和生态系统退化状 况和造成退化的原因, 从而探索共同应对这些问题 和挑战的备选方案和解决办法。此时发布的IPBES 全球评估报告受到了各界的极大重视, 全球各大媒 体纷纷报道。法国总统马克龙邀请了全球评估报告 共同主席和执行秘书介绍评估报告成果, 并做出了 关于生物多样性的新政策承诺。美国先后两次在白 宫组织召开了听证会, 探讨评估报告的主要结论。 此外, 全球评估报告还受到《生物多样性公约》等 环境协定的接受和认可。可以预见, 随着IPBES评 估报告逐渐受到各界的认可, 其影响力将逐渐提 升。

作为一个独立的政府间机制，IPBES考虑到成 员国提出的加强生物多样性科学和政策互动的有 关需求, 接受多边环境协定等机制提出的建议, 开 展了生物多样性和生态系统服务评估、支持决策、 发现知识空缺以及能力建设等工作。IPBES在国际 社会的作用不可替代, 它对了解生物多样性丧失和 生态系统服务退化的根本原因产生了重要影响, 加 深了国际社会对全球生物多样性和生态系统服务
的现状、趋势和保护措施等方面的了解，促进了全 球共识的形成, 已经成为《生物多样性公约》等相 关公约在制定未来十年行动目标的重要科学基础。

\section{4 国内参与相关工作的建议}

我国是生物多样性大国, 深入参与IPBES工作 有利于树立我国政府高度重视生物多样性保护、履 行国际义务的良好形象; 有利于学习和借鉴国际上 在该领域的经验和做法, 进一步推进我国生物多样 性保护评估与管理能力提高和队伍建设, 宣传我国 生物多样性保护成就。为切实提高我国专家的参与 力度, 提升我国的国际影响力, 针对当前存在的问 题和挑战, 在国内现有工作基础上提出如下建议。

第一, 加强科学政策互动。开展相关专题的前 期调研, 了解国内相关领域的优秀专家和整体研究 水平, 查明目前国内研究存在的空缺和短板, 结合 国内工作需求, 为国内应对指明方向。加强科学与 政策的联系, 促进科研成果在政策上的应用, 通过 凝练IPBES报告成果, 对现有成果进行优化, 针对 地方政府的实际需求, 开展地方评估, 将评估结果 转化到政策建议中, 并推广分享科学与政策相结合 的成功案例和经验。

第二, 提高专家参与能力。IPBES评估过程中 的科学性与可信度同时体现在评估作者的多元化, 这将是国内参与IPBES工作可借鉴的重要方面。邀 请不同领域专业人员参与, 通过完善专家推荐机制, 规范工作流程, 提升工作效率。除了学科多样性、 科学资格的标准外, 还需要在数据、科学、政策、 实践能力, 以及性别等方面增加遴选标准。加强科 学与政策的互动, 弥补研究领域的空缺。

第三, 加强多利益敒关方参与。IPBES已编制 并实施了一系列利益做关方参与战略, 为加强与主 要战略利益做关方的沟通和协作提供了指导, 通过 建立合作伙伴关系共同开展工作, 增进互利。为此, 建议国内提高利益做关方参与力度, 参与评估报告 的编制和审查等, 特别是不同部门、组织机构的相 关人员、有关领域的专家、从事生物多样性保护的 管理人员、决策者和政策专家, 以及掌握丰富地方 知识的专家等。

第四, 扩大宣传。加强IPBES评估报告解读, 扩 大IPBES在国内的宣传力度, 积极参与IPBES国际 
进程，加强信息共享及国际合作(张博雅等，2018), 有利于促进国内参与。此外, 包括定期编制和发布 中英文动态简报等宣传材料, 通过网络和报刊等媒 体，以国际研讨会、利益做关方对话会等形式搭建 国际国内信息共享平台，介绍中国经验，讲好中国 故事, 增加公众对IPBES的了解和广泛参与。

\section{参考文献}

Duke CS, Quach K, Jackson ST (2015) The Intergovernmental Platform on Biodiversity and Ecosystem Services (IPBES): Enhancing collaboration to support science-based decision-making, In: AGU Fall Meeting Abstracts. San Francisco, USA. https://agu.confex.com/agu/fm15/webprog ram/Paper73668.html. (accessed on 2020-06-21)

IPBES (2014a) Report of the Second Session of the Plenary of the Intergovernmental Science-Policy Platform on Biodiversity and Ecosystem Services. Antalya, Turkey. https://ipbes.net/sites/default/files/downloads/IPBES_2_17 en_0.pdf. (accessed on 2020-06-21)

IPBES (2014b) Progress Report on the Development of a Procedure for the Review of the Effectiveness of the Administrative and Scientific Functions of the Platform (deliverable 4 (e)). Bonn, Germany. https://ipbes.net/sit-es/ default/files/downloads/IPBES_3_INF_11.pdf. (accessed on 2020-06-21)

IPBES (2015) Procedure for the Review of the Effectiveness of the Administrative and Scientific Functions of the Platform (deliverable 4 (e)). Kuala Lumpur, Singapore. https://ipbes. net/sites/default/files/downloads/IPBES-4-16_EN.pdf. (accessed on 2020-06-21)

IPBES (2017a) Report of the Plenary of the Intergovernm-ental Science-Policy Platform on Biodiversity and Ecosystem Services on the Work of its Fifth Session. Bonn, Germany. https://ipbes.net/sites/default/files/ipbes-5-15_en.pdf. (accessed on 2020-06-21)

IPBES (2017b) Review of the Effectiveness of the Admin-istrative and Scientific Functions of the Platform (deliv-erable 4 (e)). Medellin, Colombia. https://ipbes.net/ sites/default/files/ipbes-6-10-en.pdf. (accessed on 2020-06-21)
IPBES (2019a) Review of the Effectiveness of the Administrative and Scientific Functions of the Platform. Paris, France. https://ipbes.net/sites/default/files/ipbes-7-5_ en_review.pdf. (accessed on 2020-06-21)

IPBES (2019b) Report of the Plenary of the Intergovernmental Science-Policy Platform on Biodiversity and Ecosystem Services on the Work of Its Seventh Session, Paris, France. https://ipbes.net/sites/default/files/ipbes-7-10_en.pdf. (accessed on 2020-06-21)

Ma KP (2012) Intergovernmental Science-Policy Platform on Biodiversity and Ecosystem Services (IPBES): An IPCC for biodiversity. Biodiversity Science, 20, 409-410. (in Chinese) [马克平 (2012) IPBES: 生物多样性领域的 IPCC. 生物多样性, 20, 409-410.]

Pan YX, Zhang BY, Wu Y, Dai FB, Tian Y (2020) The latest developments of IPBES and China's countermeasures. Biodiversity Science, 28, 1286-1291. (in Chinese with English abstract) [潘玉雪, 张博雅, 吴杨, 戴逢斌, 田瑜 (2020) IPBES工作进展及我国对策建议. 生物多样性, 28, 1286-1291.]

Tian Y, Li JS, Lan CZ, Li XS (2015) Interpretation of the work programme of Intergovernmental Science-Policy Platform on Biodiversity and Ecosystem Services for the period 2014-2018. Biodiversity Science, 23, 543-549. (in Chinese with English abstract) [田瑜, 李俊生, 兰存子, 李秀山 (2015) 生物多样性和生态系统服务政府间科学-政策平 台2014-2018年工作方案解析. 生物多样性, 23, 543-549.]

Tian Y, Lan CZ, Xu J, Li XS, Li JS (2016) Assessment of pollination and China's implementation strategies within the IPBES framework. Biodiversity Science, 24, 1084-1090. (in Chinese with English abstract) [田瑜, 兰存子, 徐靖, 李秀 山, 李俊生 (2016) IPBES框架下的全球传粉评估及我国 对策. 生物多样性, 24, 1084-1090.]

Zhang BY, Pan YX, Xu J, Tian Y (2018) IPBES thematic assessment on land degradation and restoration and its potential impact. Biodiversity Science, 26, 1243-1248. (in Chinese with English abstract) [张博雅, 潘玉雪, 徐靖, 田 瑜 (2018) IPBES土地退化和恢复专题评估报告及其潜在 影响. 生物多样性, 26, 1243-1248.]

(责任编委: 薛达元 责任编辑: 时意专) 